\title{
Mobility Management Requirements for Diverse 5G Wireless Networks
}

\author{
Saba Qasim Jabbar \\ School of Electronic Information and Communication \\ Engineering, Huazhong University of Science and \\ Technology, Wuhan 430074, P.R. China \\ E-mail: 7@yahoo.com
}

\author{
Dheyaa Jasim Kadhim \\ Electrical Engineering Department, University of Baghdad, \\ Baghdad, Iraq \\ E-mail: 7@yahoo.com
}

\author{
Raed Abduljabbar Aljiznawi, Naseer Hwaidi Alkhazaali \\ School of Electronic Information and Communication Engineering, Huazhong University of Science and Technology, Wuhan \\ 430074, P.R. China \\ Ministry of Communication, Iraqi Telecommunication and Post Company, Baghdad, Iraq \\ E-mail: 7@yahoo.com
}

\begin{abstract}
Mobile users increased demand for access to mobile communication services is accelerating the technological development towards the integration into the various modes of wireless access communications with respect to coverage, $\mathrm{QoS}$ assurance, implementation, operational costs supported features, etc. The integration should take into account the user mobility from one access point to another. In diverse 5G wireless networks, mobility management provide mobile users with continuously get the connection when they move among different subnets based on their service needs. With this heterogeneity, users will be able to choose radio access technology that offers higher quality, data speed and mobility which is best suited to the required multimedia applications with the best performance and minimum cost. It is necessary to ensure that the internet application efficient state is maintained while used diverse $5 \mathrm{G}$ wireless networks. This is one of motivation for conducting this work.
\end{abstract}

Keywords-G networks; obility management; ndoff management; cation management

\section{INTRODUCTION}

During the past three decades, the world has seen significant changes in the wireless Telecommunications industry. There have been some remarkable aspects to the rapid growth in wireless communications, as seen by the large expansion in mobile systems [1]. Wireless systems consist of Wireless Wide-Area Networks (WWAN), Wireless Local Area Networks (WLAN) and Wireless Personal Area Networks (WPAN). The rapid growth in the number of wireless applications, services and devices using a single wireless technology such as a second generation (2G) and third generation $(3 \mathrm{G})$ wireless system would not be efficient to deliver high speed data rate and quality-of-service (QoS) support to mobile users in a seamless way. The current generation wireless systems (sometimes referred to as Fourth generation $(4 \mathrm{G})$ systems) are being devised with the vision of heterogeneity in which a mobile user/device will be able to connect to multiple wireless networks (e.g., WLAN, cellular, WMAN) simultaneously [1][2].

Diverse 5G wireless systems will achieve efficient wireless resource utilization, seamless handoff, global mobility with QoS support through load balancing and tight integration with services and applications in the higher layers. After all, in such a diverse wireless access network, a mobile user should be able to connect to the Internet in a seamless manner. The wireless resources need to be managed efficiently from the service provider's point of view for maximum capacity and improved return on investment. Seamless roaming or mobility management is crucial to ubiquitous computing and requires network management operations to avoid service degradation. Both location management and handoff management constitute mobility management [3][4].

Many researchers wrote on the scope of heterogeneous networks, seamless mobility and vertical handoff some of them wrote on the role of it to improve the network performance and others wrote on the field of optimizing its works. Mark stemm in [5] has explored methods which enable seamless mobility in wireless LAN networks with using 802.11 networks configured to work like a single umbrella network. He established mathematical relationship that allows a vertical handoff. Zahran [6][7] studied the performance of vertical handoff using the integration of heterogeneous networks in $3 \mathrm{G}$ cellular and wireless local area networks with MIP supported using loosely-coupled architectures. The results showed that the adaptive VHO approach was a good improvement in the system resource and that the proposed analytical model provided design guidelines for the vertical handoff in the next generation integrated wireless networks. There are several benefits to a diverse $5 \mathrm{G}$ wireless network as opposed to a traditional Uniform wireless network including increased reliability, improved spectrum competence, improved the quality of service and increased network coverage. Reliability is also enhanced because when one particular radio access technology within the diverse wireless network fails, it may still be gifted to maintain a correlation by falling back to another [8].

The remainder of the paper is organized as follows: Section 2 discusses the mobility management in diverse $5 \mathrm{G}$ wireless networks while Section 3 submits the important requirements for mobile management of our proposed 
scheme. Section 4 presents our proposed scheme for mobility management among different $5 \mathrm{G}$ wireless networks. Section 6 evaluates the routine of our proposed scheme in several important metrics and compares the results with those of the other approach. Section 5 draws concluding remarks and directions for future work.

\section{MOBILITY MANAGEMENT OF DIVERSE 5G WIRELESS NETWORKS}

Seamless roaming or mobility is crucial to ubiquitous computing and requires network management operations to avoid service degradation. Both location management and handoff management constitute mobility management. Location management involves two processes. The first process is called location registration, or location update, in which the MT periodically informs the network of its current location, which leads the network to authenticate the user and update its location profile in a database [9]. The second process related to location management is called paging. The handoff management is one of the major functions under mobility management that embraces several aspects of user mobility and mobility support procedures for wireless networks. Handoff management includes wireless terminal handoff management considerations within one network called horizontal handoff and handoff management across different wireless networks which could be based on different wireless access technologies termed vertical handoff.

\section{A. Mobility Management Functions}

Mobility management generally deals with automatic roaming, authentication, and intersystem handoff. Automatic roaming includes a set of network functions that allow a subscriber to obtain service outside the home service provider area. These functions are automatic and do not require special subscriber actions. The automatic roaming functions are divided into:

- Mobile station (MS) service qualification

- MS location management

- MS state management

- Home location register (HLR), and VLR fault recovery

- The authentication process requires that end users of the system are authenticated i.e., the identity of each subscriber is verified.

\section{B. Typical Requirements of Mobility Management}

There are some important and typical requirements to provide seamless roaming between diverse wireless networks, these requirements are such as:

- When an interface loses contact, re-establishment attempt should be performed automatically.

- A handoff between different network interfaces could occur due to various reasons such as poor signal or a user wanting more facilities and thus moving to a different network.

- Handoff delay and packet loss should be minimized, especially in real time applications.
- If a situation occurs wherein the session is not maintained throughout or is interrupted by varying network conditions, the session will be terminated and re-initiated if the user is interested in continuing the session through a different interface.

\section{Handoff Across Diverse $5 g$ Wireless Networks}

Handoff management is one of the mobility management components; it maintains mobile nodes connections active while roaming or switching into a new area. Depending on the type of attachment, the handoff can be classified into two types: horizontal handoff and vertical handoff. In the horizontal handoff, the mobile terminal will not change the technology deployed for its connection even when moving from one point of attachment to another. However, in the vertical handoff, the mobile terminal (MT) will change the technology when handing off and when moving from one point of attachment to another.

The handoff process is divided into three phases [10]: Network Discovery, Handoff Decision and Handoff Implementation. Periodically the system monitors for a better network which the mobile terminal can be handed off. The handoff considerations include several different criteria depending on the algorithms and the goals set for handoff. During the system discovery phase, the mobile terminal determines which networks can be used. These networks may also advertise the supported data rates and Quality of Service (QoS) parameters [11]. The handoff decision uses an algorithm that optimizes based on a selected set of criteria to decide when to handoff. The decision is very crucial and several different interesting solutions were proposed to address the problem [12].In vertical handoff decision phase, the mobile terminal determines whether the connections should continue using the current network or be switched to another network. The decision may depend on various parameters or metrics including the type of the application (e.g., conversational, streaming), minimum bandwidth and delay required by the application, access cost, transmit power, and the user's preferences. During the vertical handoff execution phase, the connections in the mobile terminal are re-routed from the existing network to the new network in a seamless manner. This phase also includes the authentication, authorization, and transfer of a user's context information [13].Thus vertical handoffs are implemented across heterogeneous cells of access systems, which are differ in several aspects such as bandwidth, data rate, frequency of operation, and better QoS etc [14].

\section{MOBILITY REQUIREMENTS FOR PROPOSED SCHEME}

In order to satisfy seamless mobility a combining vertical handoff algorithm was proposed with some modification using adaptive lifetime and relative received signal algorithms. The topology model used in this study is the overlapping of heterogeneous WLAN and Cellular networks. Different scenarios are used to visualize two mobile users 
with a supporting the Mobile IP assumed for mobility management purpose across the two access networks. There are proposed requirements for vertical handoff in diverse $5 \mathrm{G}$ wireless network can be summarized as follows; (Bandwidth, Handoff Latency, Power Consumption, Network Cost, User Preferences, Throughput, Velocity).

Because of the overlaid architecture of heterogeneous networks, handing off to an embedded network, having small cell area, travelling at high speeds is discouraged since a handoff back to the original network would occur very shortly afterwards. The dynamic requirements include RSS, velocity, throughput, user preferences as parameters and non-dynamic requirements include network cost, power consumption, network security and bandwidth parameters. A good handoff mechanism decision model should have both dynamic and non-dynamic metrics.

\section{PROPOSED SCHEME FOR MOBILITY MANAGEMENT}

This work focus develops a vertical handoff decision scheme for optimizing the efficiency of vertical handoffs in heterogeneous wireless networks. So the performance of the vertical handoff algorithm was evaluated under two different approaches: the first approach is called forward procedure for the moving of Mobile Terminal MT's from Wireless Local Area Network (WLAN) to Cellular Network $(\mathrm{CN})$ and the second is called the reverse procedure for moving of MT from CN to WLAN. In this work, a new hybrid algorithm is proposed to evaluate the performance of vertical handoff process. the following subsections with show how these two procedures are working.

\section{A. Forward Procedure for Vertical Handoff}

Now, discuss and analyze two main scenarios in VHO : MI and MO that represent the MT Moving into the preferred network and Moving Out of the preferred network, respectively. When the MT is connected to the Cellular network realizing that a WLAN is available (moving in) within an overlapped area by $\mathrm{CN}$, the following steps occur in MI scenario:

- MT's home network is the $\mathrm{CN}$ as a first case. Then MT detects a WLAN AP beacon which is in feasible range.

- The HO algorithm decides or the MT decides to handoff to WLAN and it starts with a set of L1/L2 handoff procedures

- MT sends a standby message to the home network to prevent the $\mathrm{CN}$ detaches the MT when it is moving.

- Then, MT can access the IP network and sends an Agent Solicitation to locate FA (foreign agent), FA replies to the MT.

- The MT can send Registration Request to it is HA (home agent). After updating the address in the HA, the downward $\mathrm{HO}$ occurs.

\section{B. Reverse Procedure for Vertical Handoff}

In the reverse situation, when the MT moves away from the coverage of WLAN within the Cellular coverage, (moving out), it can be illustrate that the following steps occur in MO scenario:

- Signal received from the Access Point (AP) in the WLAN is strong; represents HA, and then becomes weak;

- MT decides or the network decides to handoff to a $\mathrm{CN}$ if there is no another AP available and it starts with a set of handoff procedures.

- Start/update Mobile IP: Foreign Agent (FA) in the MT gets activated using the Mobile IP protocol and MT uses new IP address by using the second part of MT, the universal subscriber identity mobile (USIM).

- If the MT did not attach the network or activate the session before, it should attach the network and activate a session before getting the service.

- The Home Agent (HA) in the WLAN is informed about the new IP address through the Mobile IP protocol and the upward VHO is occurring.

- The HA encapsulates and tunnels any packets arriving for the MT to the FA of the CN.

\section{PERFORMANCE ANALYSIS OF PROPOSED SCHEME}

Since Mobile-IP provides only one IP tunnel, the MT can connect to one network only at a time. The MT periodically scans (discovers), the available interfaces and measures the relative RSS When using high accuracy, the best access network achieving that matches the predefined vertical handoff algorithm. The suggested scheme for vertical handoff was tested and evaluated using MATLAB R2012a simulation for applying in two procedures. Two scenarios $\mathrm{MO}$ and MI were evaluated using these two procedures. Thus, the average number of vertical handoff verses mobile terminal velocity were calculated and compared with traditional handoff algorithm using RSS and threshold with hysteresis level. The results shown in Fig. 1 illustrate the decreasing number of vertical handoff in hybrid algorithm in comparison with traditional algorithm. That explains the effect of selecting the type and size of window. In Figs. 2 and 3 , the effects of adaptive signal strength threshold was studied using the combining procedures, three different values of ASST were used and the results shows that the increase of ASST is the decrease of the average number of vertical handoff. In Fig. 4, we see that the percentage between our algorithm and the traditional algorithm in the number of vertical handoff decreased. The percentage is about (62-37)\% in linear trend line of decreasing. We show that the level of decreasing is reduced when the velocity becomes high. This can be concluded from this figure. This algorithm can be satisfied and used in low user mobile for more attention. 


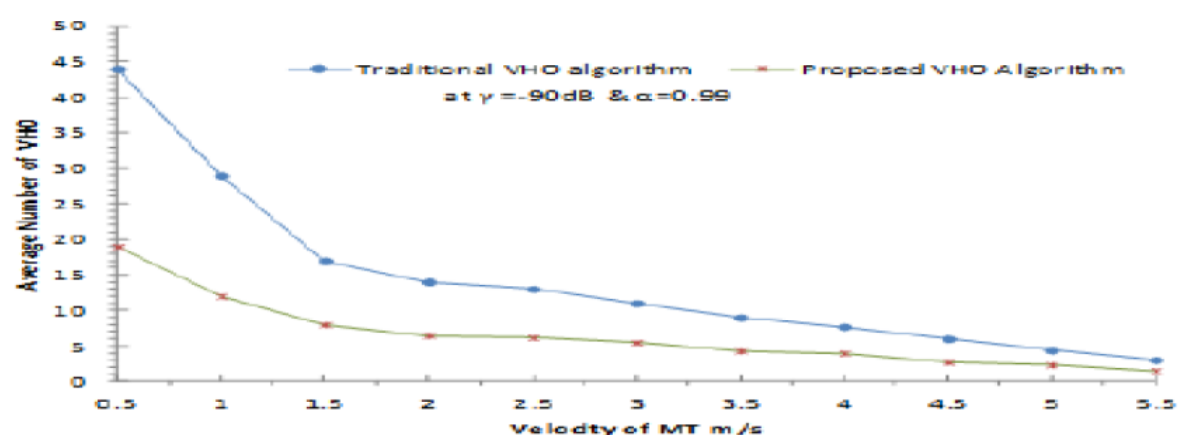

Figure 1. Comparison between two procedures of proposed scheme

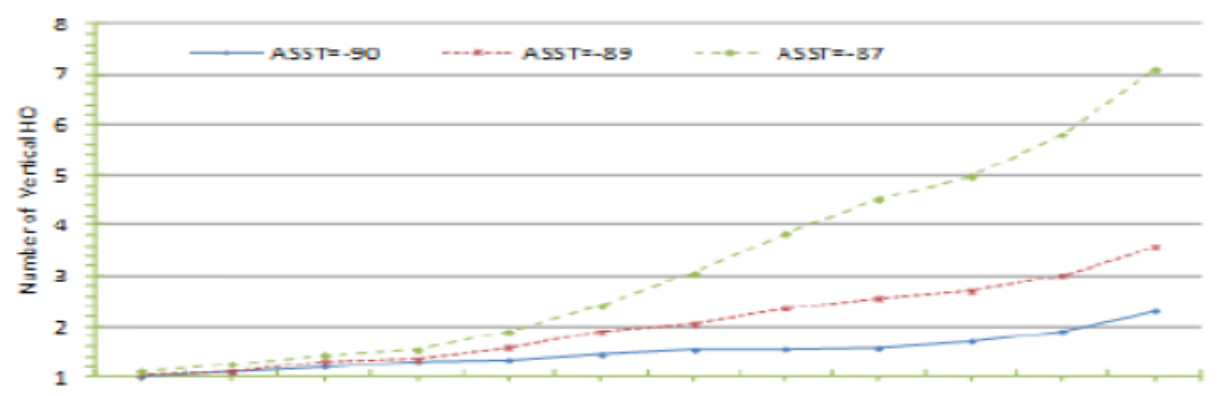

Figure 2. Number of vertical handoffs vs. velocity

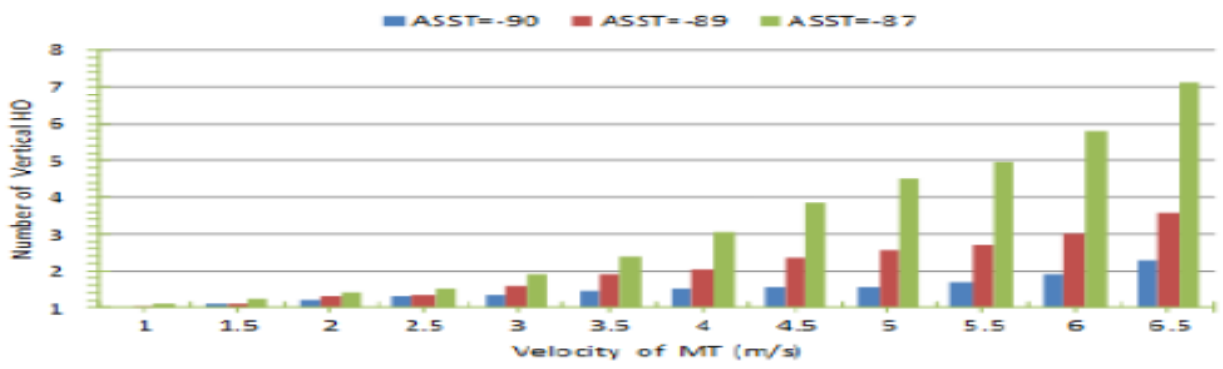

Figure 3. Number of handoffs vs. velocity at different ASST

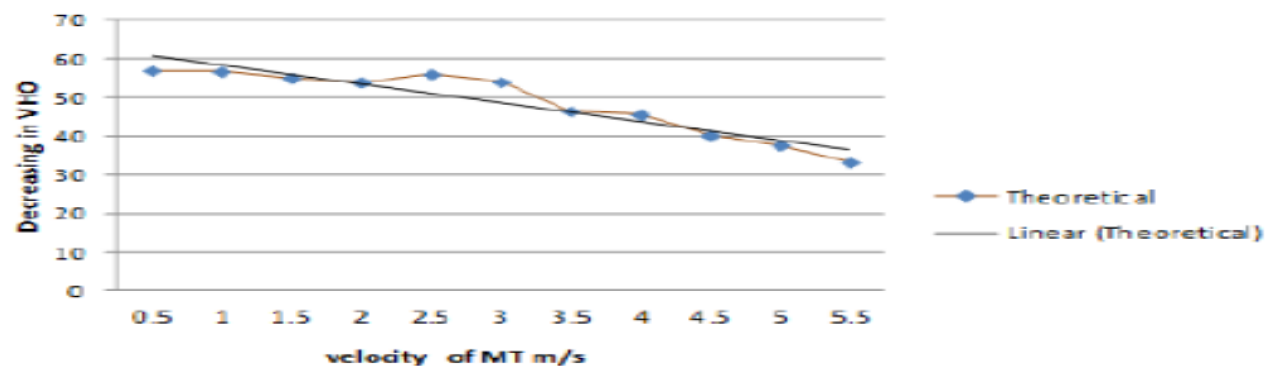

Figure 4. Decreasing percentage of proposed scheme for two procedures

\section{CONCLUSIONS}

The work of paper has two mobility procedures. In these procedures, the performance of the vertical handoff algorithm was evaluated for the moving of Mobile Terminal MT's from Wireless Local Area Network (WLAN) to Cellular Network $(\mathrm{CN})$ and the inverse movement of MT's moving from $\mathrm{CN}$ to WLAN. In this work, a new hybrid algorithm is proposed to evaluate the performance of vertical handoff process. According to the results in previous section, the suggested hybrid vertical handoff algorithm show that the increasing of dependency on the WLAN gave and provided the optimal system resource, as well as keeping the resources of the heterogonous network for users located outside the WLAN. The value of ASST is an important factor that determines the number of handoff.

\section{REFERENCES}

[1] G. L. Stuber, Principles of Mobile Communication, 3rd and 4th Edition, Springer Science Business Media LLC. 2002, 2011.

[2] A. Dutta, "Systems Optimization for Mobility Management", Dissertation, Columbia University, 2010. 
[3] X. Yan, Y. A. Sekerciog, "A survey of vertical handover decision algorithms in Fourth Generation heterogeneous wireless networks", Elsevier B.V., pp.1848-1863, 2010.

[4] E. Hossain, Heterogeneous Wireless Access Networks, Architectures and Protocols, Springer Science and Business Media LLC, 2008.

[5] M. Stemm "Vertical Handoff in Wireless Overlay Networks", international symposium on mobility managements and wireless access, Vol.7, No.12, 2006.

[6] B. Liang, A. H. Zahran, and A. Saleh, "Application Signal Threshold Adaptation for Vertical Handoff in Heterogeneous Wireless Networks", IFIP International Federation for Information, pp. 1193-1205, 2005.

[7] B. Liang, A. H. Zahran and Aladdin Saleh, "Signal Threshold Adaptation for Vertical Handoff in Heterogeneous Wireless Networks", Mobile Network Application, Springer, pp. 625-640, 2006.

[8] T. A. Yahiya, H. Chaouch, "An Optimized Handover Decision for Heterogeneous Wireless Networks ", ACM, pp. 137-142. 2009.
[9] M. Syuhadal, M. Firuz, "Performance Evaluation of Vertical Handoff in Fourth Generation (4G) Networks Model", IEEE. pp. 392-398. August 2008.

[10] S. E. Kim, J. A. Copeland, "TCP for Seamless Vertical Handoff", IEEE Global Telecommunications Conference, Vol. 2 pp. $661-665$, 2003.

[11] M. L. Alkhayat, "Virtual Wireless Network Service Design and Evaluation", Dissertations, Louisville University, Kentucky, 2009.

[12] F. Xu, L. Zhang, and Z. Zhou, "Interworking of WiMAX and 3GPP Networks Based on IMS," IEEE Communications Magazine, vol. 45, pp. 144-150. 2007.

[13] F. Zhu and J. McNair, "Optimizations for Vertical Handoff Decision Algorithms" IEEE Communications Society, WCNC, Vol. 2, pp. 867-872. 2004

[14] D. Sarddar, S. Maity, A. Raha and R. Jana, "A RSS Based Adaptive Handoff Management Scheme in Heterogeneous Networks" IJCSI International Journal of Computer Science Issues, Vol. 7, Issue 6, pp. 232-238, Nov. 2010. 